\title{
Role of Adenosine in the Sympathetic Activation Produced by Isometric Exercise in Humans
}

\author{
Fernando Costa and Italo Biaggioni \\ Division of Clinical Pharmacology, Departments of Medicine and Pharmacology, Vanderbilt University, Nashville, Tennessee $37232-2195$
}

\begin{abstract}
Isometric exercise increases sympathetic nerve activity and blood pressure. This exercise pressor reflex is partly mediated by metabolic products activating muscle afferents (metaboreceptors). Whereas adenosine is a known inhibitory neuromodulator, there is increasing evidence that it activates afferent nerves. We, therefore, examined the hypothesis that adenosine stimulates muscle afferents and participates in the exercise pressor reflex in healthy volunteers. Intraarterial administration of adenosine into the forearm, during venous occlusion to prevent systemic effects, mimicked the response to exercise, increasing muscle sympathetic nerve activity (MSNA, lower limb microneurography) and mean arterial blood pressure (MABP) at all doses studied (2, 3, and $4 \mathrm{mg})$. Heart rate increased only with the highest dose. Intrabrachial adenosine (4 mg) increased MSNA by $96 \pm 25 \%(n=6, P<0.01)$ and MABP by $12 \pm 3 \mathrm{mmHg}(P<0.01)$. Adenosine produced forearm discomfort, but equivalent painful stimuli (forearm ischemia and cold exposure) increased MSNA significantly less than adenosine. Furthermore, adenosine receptor antagonism with intrabrachial theophylline $(1 \mu \mathrm{g} / \mathrm{ml}$ forearm per min) blocked the increase in MSNA $(92 \pm 15 \%$ vs. $28 \pm 6 \%, n=7, P<0.01)$ and MABP (38 \pm 6 vs. $27 \pm 4 \mathrm{mmHg}, P=0.01)$ produced by isometric handgrip ( $30 \%$ of maximal voluntary contraction) in the infused arm, but not the contralateral arm. Theophylline did not prevent the increase in heart rate produced by handgrip, a response mediated more by central command than muscle afferent activation. We propose that endogenous adenosine contributes to the activation of muscle afferents involved in the exercise pressor reflex in humans. (J. Clin. Invest. 1994.93:1654-1660.) Key words: adenosine $\bullet$ theophylline $\bullet$ isometric exercise • muscle sympathetic nerve activity
\end{abstract}

\section{Introduction}

Isometric exercise sets in motion a series of events that work in tandem to activate the sympathetic nervous system and increase blood pressure. The cortical impulses that initiate voluntary muscle contractions (the so-called central command) contribute to the increase in sympathetic tone. Local mechanisms also play a pivotal role in the triggering of this reflex. Muscle contraction activates afferent nerves sensitive to mechanical

Dr. Italo Biaggioni, Clinical Research Center, AA3228 MCN, Vanderbilt University, Nashville, TN 37232-2195.

Received for publication 12 July 1993 and in revised form 17 December 1993.

J. Clin. Invest.

(c) The American Society for Clinical Investigation, Inc.

0021-9738/94/04/1654/07 \$2.00

Volume 93, April 1994, 1654-1660 deformation ("mechanoreceptors"). The increase in muscle metabolism and the relative ischemia that results from compression of blood vessels by the contracting muscle generate metabolic products that then activate chemo-sensitive afferent nerves ("chemoreceptors" or "metaboreceptors"). These chemoreceptors constitute the afferent limb of a reflex that results in sympathetic activation and increased blood pressure. Our understanding of this reflex in humans has been improved by the ability to measure muscle sympathetic nerve activity (MSNA) ${ }^{1}$ directly using microneurographic techniques(1). Using this approach it has been shown that voluntary isometric handgrip increases sympathetic nerve activity and blood pressure in humans, in large part due to stimulation of these forearm afferents (1).

Adenosine is known to be produced during increased metabolic demands and, in particular, during muscle contraction and ischemia (2). It could be proposed, therefore, that adenosine accumulates during isometric exercise and activates muscle afferents. In agreement with this hypothesis, there is growing evidence that adenosine excites a variety of afferent nerves leading to sympathetic activation (3). Adenosine activates renal afferents (4), myocardial afferents (5), and carotid body and arterial (6) chemoreceptors. Activation of afferent nerves by adenosine appears to be particularly prominent in humans, and may explain most of the cardiovascular and respiratory effects of intravenous adenosine in this species.

Given the excitatory effect of adenosine on other afferent fibers, the purpose of the present studies was to test the hypothesis that adenosine activates the muscle afferents involved in the exercise pressor reflex. Towards this aim, we have used two complementary approaches. First, we determined if the effects of exogenous adenosine, acting locally in the forearm, mimic those produced by the exercise pressor reflex. For these studies we administered increasing doses of adenosine via the brachial artery while the venous circulation of the arm was occluded, to avoid spillover of adenosine into the systemic circulation. Second, we determined if blockade of endogenous adenosine would alter the exercise pressor reflex. For these studies, the adenosine receptor antagonist theophylline was administered via the brachial artery to minimize systemic effects. Isometric exercise was performed during intrabrachial administration of saline or theophylline while monitoring the heart rate and blood pressure responses. Concomitant measurements of sympathetic nerve activity were made in the lower limb, through an electrode placed in the peroneal nerve, as an assessment of the systemic sympathetic activation produced by exercise.

\section{Methods}

We studied a total of 19 normal, healthy volunteers of both genders, 19-44 yr old (mean, $25 \pm 2 \mathrm{yr}$ ). Subjects were nonsmokers, who were

1. Abbreviation used in this paper: MSNA, muscle sympathetic nerve activity. 
free of medications and had abstained from methylxanthines for at least $72 \mathrm{~h}$ before the study day. The protocol was approved by the Vanderbilt University Institutional Review Board. Volunteers were informed of the characteristics of the study and gave written consent.

Instrumentation. Subjects were studied fasted and in the supine position. Heart rate was monitored by surface electrocardiography coupled to a rate computer. Arterial blood pressure was measured through an indwelling catheter placed in the left brachial artery and connected through two three-way valves to a pressure transducer. The second and third ports were used for intraarterial drug administration. Cardiovascular signals were modulated on signal conditioners and displayed on a thermal array recorder (TA2000; Gould Inc., Cleveland, OH). To measure expiratory volume, the subjects breathed through a mouthpiece, and inspiration and expiration were isolated with one-way valves. Expiratory flow was measured with a heated pneumotachograph (Fleisch pneumotach; Gould Inc.) connected to a differential transducer (Validyne Engineering Corp., Northridge, CA). Expiratory volume was measured by integrating the expiratory flow signal over time (integrator signal conditioner, Gould Inc.).

Sympathetic nerve activity was measured as previously described (7). The approximate location of the right peroneal nerve at the level of the fibular head was determined by transdermal electrical stimulation (10-60 V, 0.01-ms duration), which produced painless muscle contractions of the foot. A tungsten needle electrode, with a shaft diameter of $200 \mu \mathrm{m}$ and an uninsulated tip diameter of $1-5 \mu \mathrm{m}$, was inserted into the nerve. A similar electrode with a larger uninsulated tip was inserted subcutaneously near the recording electrode to serve as a reference electrode. The recording electrode was positioned within the nerve to obtain multiunit recordings of sympathetic efferent activity. Placement of the electrode was guided by electrical stimulation (1-4 V, 0.01-ms duration), which produced muscle twitches of the foot but not paresthesias. Electrical stimulation was done with a stimulator connected to an isolation unit (S88 and SIU8TB; Grass Instruments, Quincy, MA). The electrode was then switched to a recording mode and fine adjustments of its position were made to obtain a satisfactory recording site as described below. Recorded signals were fed to a preamplifier (gain, $\times 1,000$ ) and were filtered using a band width between 700 and 2,000 $\mathrm{Hz}$. The filtered signal was rectified, amplified (gain, $\times 100$ ), and integrated in a resistance-capacitance network using a time constant of 0.1 (Nerve Traffic Analysis System 662C-3; University of Iowa Bioengineering, Iowa City, IA). The final signal was monitored using a storage oscilloscope (S111A; Tektronics, Beaverton, OR) and recorded after fourfold amplification (TA-2000 recorder; Gould Inc.)

Criteria for an adequate muscle sympathetic nerve activity recording were as follows: $(a)$ electrical stimulation produced muscle twitches, but no paresthesias; $(b)$ stretch of the tendons in the foot evoked proprioceptive afferent signals, whereas cutaneous stimulation by slight stroking of the skin did not; $(c)$ held expiration increased neural activity at a site where arousal stimuli did not; and $(d)$ nerve activity increased during the hypotensive phase of the valsalva maneuver and was suppressed after the release of valsalva during blood pressure overshoot. Muscle sympathetic nerve activity was also monitored throughout the study using a loudspeaker. This helped in the identification of potential artifacts, such as electrostatic discharges, which were also identified in the integrated neurogram by their rapid onset and shorter duration.

Study protocols. Subjects were allowed to rest in a quiet room for 20-30 min after instrumentation to ensure that all cardiovascular and microneurographic parameters had returned to resting values. The subjects were warned ahead of time of all procedures done, and that they may produce discomfort, in order to avoid anticipatory reactions. Intraarterial bolus injections of either saline or increasing doses of adenosine $(2,3$, and $4 \mathrm{mg})$ were then administered in a single-blinded fashion while monitoring the subject's heart rate, blood pressure, and muscle sympathetic nerve activity. Intraarterial adenosine was administered during simultaneous occlusion of the venous circulation to prevent its spillover into the systemic circulation. For this purpose a pneumatic cuff was placed proximal to the injection site and inflated to
$50 \mathrm{mmHg}$ immediately before injection, and was maintained inflated for the $2 \mathrm{~min}$ after the adenosine injection. The highest dose of adenosine $(4 \mathrm{mg}$ ) was also given intravenously and intraarterially without occluding the venous circulation in order to examine the systemic effects of adenosine, and to determine the effectiveness of venous occlusion in avoiding these systemic effects. Sufficient time was allowed between injections to allow parameters to return toward baseline values.

Intraarterial administration of adenosine has been reported to produce forearm pain (8). It is possible, therefore, that activation of sensory afferents may contribute to the changes in the MSNA produced by intrabrachial adenosine. To determine this potential contribution we compared the effects of two painful stimuli, ischemia and cold exposure, to those produced by intrabrachial adenosine. For this purpose, saline and increasing doses of adenosine $(0.5-4 \mathrm{mg})$ were administered intrabrachially in a single-blinded fashion during venous occlusion. Subjects were asked to rank the intensity of pain on a scale from 0 (no pain) to 10 (excruciating pain) after each dose. Forearm ischemia of increasing duration $(1,2,3$, and $5 \mathrm{~min})$ was induced in the same subjects by inflating a cuff placed around the arm to $50 \mathrm{mmHg}$ above systolic blood pressure. The effects of cold exposure were determined by sequentially applying one pack of ice $(140 \times 130 \mathrm{~mm})$ over the forearm, followed by two packs of ice over the forearm, and finally by placing the hand in ice water for $1 \mathrm{~min}$. The subjects were asked to rank the intensity of the pain at the end of each period of ischemia or cold exposure, as described above. We paired the dose of adenosine in each subject with the duration of ischemia or the extent of cold exposure that produced similar intensities of pain. We then compared the effect of those paired stimuli on MSNA.

A second group of volunteers was studied to determine the role of endogenous adenosine in the exercise pressor reflex. We used isometric handgrip to elicit an exercise pressor reflex, and theophylline as an adenosine receptor antagonist. Subjects were instrumented as described above and allowed to rest in a quiet room for 20-30 min. Saline was then infused into the left brachial artery at the same rate of infusion calculated for theophylline (see below). After $5 \mathrm{~min}$ of saline infusion, we performed three experimental protocols in the following sequence: (a) Handgrip followed by ischemia. Volunteers were asked to perform a sustained handgrip for $3 \mathrm{~min}$ at $30 \%$ of maximal voluntary contraction, using a handgrip dynamometer (Lafayette Instrument Co., Lafayette, IN). A pneumatic cuff on the upper arm was inflated to $50 \mathrm{mmHg}$ above the systolic blood pressure immediately after release of the handgrip and was maintained for $2 \mathrm{~min}$ (posthandgrip ischemia). (b) Control handgrip. Static isometric exercise was performed at $30 \%$ of maximal voluntary contraction for $3 \mathrm{~min}$ in the right arm. (c) Simultaneous handgrip and ischemia. Circulatory arrest was induced by inflating a pneumatic cuff, placed in the upper left arm, to $50 \mathrm{mmHg}$ above the systolic blood pressure. The subject was simultaneously asked to perform a sustained handgrip of the left hand at $30 \%$ of maximal voluntary contraction. Both exercise and ischemia were maintained for $2 \mathrm{~min}$.

After this experimental protocol, the infusion of saline was replaced by an infusion of aminophylline $(2.5 \mathrm{mg} / \mathrm{ml})$ into the left brachial artery at a rate of $1 \mu \mathrm{g} / \mathrm{ml}$ of forearm volume/min. This dose has been previously shown to produce negligible systemic effects (9). 5 min after starting the aminophylline infusion the exercise protocols described above were repeated in the same sequence. Heart rate, blood pressure, and MSNA were monitored throughout the study. Blood pressure was monitored continuously either through the left brachial catheter or with an automated device placed in the right arm (Finapres 2300; Ohmeda, Englewood, $\mathrm{CO}$ ) during the periods of circulatory arrest of the left arm. Blood samples were drawn from the antecubital vein of both arms at the end of the study for measurement of plasma theophylline levels.

Drugs, analytical methods, and statistical analysis. Adenosine was purchased from Sigma Chemical Co. (F\&D Division, St. Louis, MO) and dissolved in normal saline at a concentration of $6 \mathrm{mg} / \mathrm{ml}$. The solution was tested for sterility and pyrogenicity. Aminophylline ( 25 
$\mathrm{mg} / \mathrm{ml}, 85 \%$ anhydrous theophylline) was purchased from American Regent Laboratories, Inc. Shirley, NY.

Mean arterial blood pressure was calculated as two-thirds of the diastolic pressure plus one-third of the systolic pressure. Measurements of muscle sympathetic nerve activity were made from the original tracings of the mean voltage neurograms using a digitizer tablet coupled to Sigma Scan Software (Jandel Scientific, Corte Madera, CA) in a microcomputer. The amplitude of each "burst" was measured in millimeters and total activity was defined as the sum of "burst" amplitude over a 60 -s period and expressed in arbitrary units. The effect of a given intervention (e.g., drug administration) on muscle sympathetic nerve activity was expressed as percent change from the resting period preceding the intervention.

Theophylline was measured by high performance liquid chromotography as described previously (10). Data were analyzed using the Number Cruncher Statistical System software (NCSS, Kaysville, Utah) in a microcomputer. Statistical analysis was performed using $t$ test for single comparisons, and analysis of variance (ANOVA) for multiple comparisons. Post hoc tests (Duncan's) were used to determine individual differences only if significant group differences were found with ANOVA. Values of $P \leq 0.05$ were considered significant. Results are expressed as mean \pm SEM.

\section{Results}

Effects of intrabrachial administration of adenosine. The effects of bolus injections of adenosine into the antecubital vein and into the brachial artery during simultaneous venous occlusion are compared in Fig. 1. Intravenous adenosine produced a biphasic blood pressure response as reported previously (11), with an initial increase followed by a decrease in blood pressure. The pressor phase was accompanied by an increase in minute ventilation and an initial increase in MSNA followed by a temporary suppression in MSNA. The depressor phase
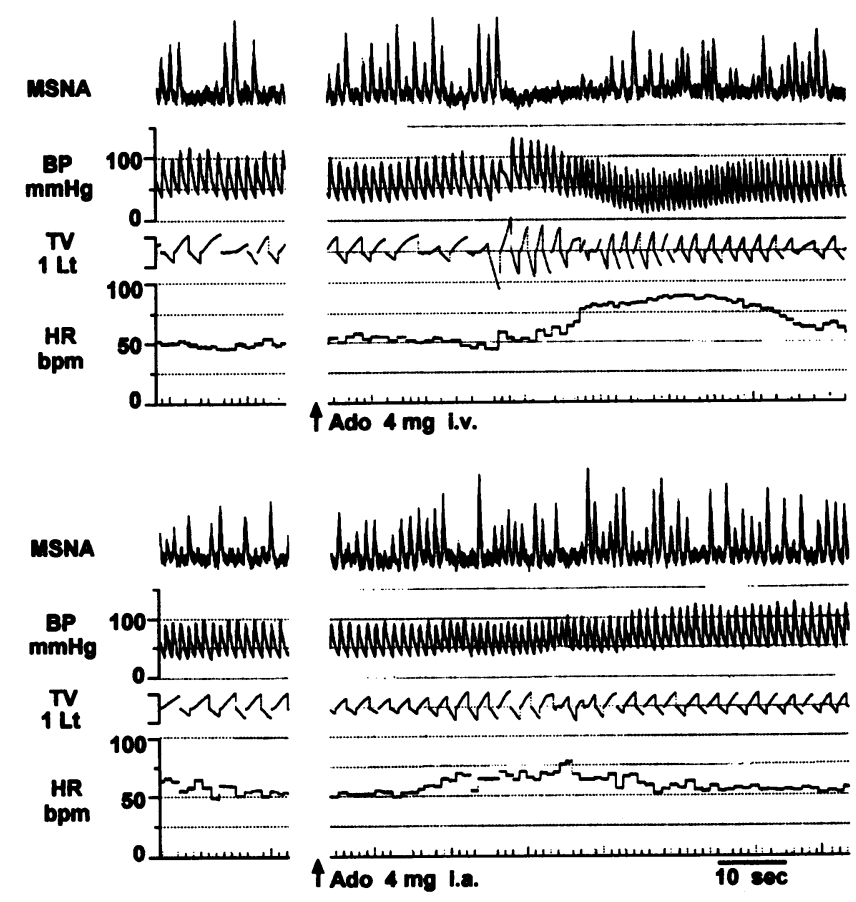

Figure 1. Tracing shows recordings of integrated neurograms of muscle sympathetic nerve activity $(M S N A)$, blood pressure $(B P)$, tidal volume $(T V)$, and heart rate $(H R)$ at baseline, and after bolus injection of $4 \mathrm{mg}$ adenosine when administered either intravenously (top), or intraarterially during simultaneous venous occlusion (bottom).
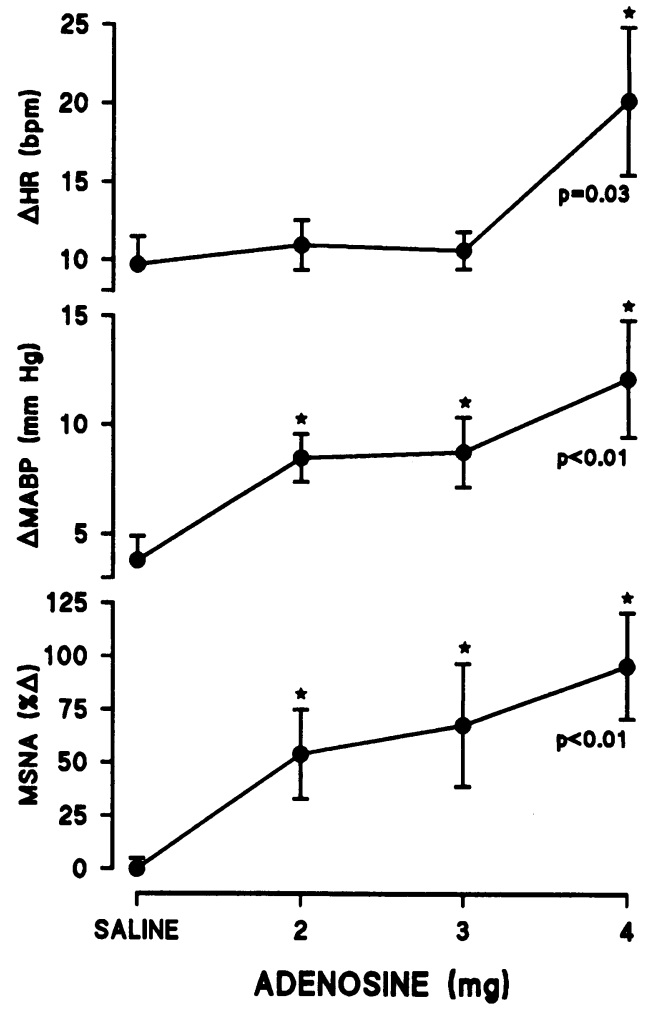

Figure 2. Cardiovascular and sympathetic effects of intrabrachial adenosine. Line graphs show the effects of saline and increasing intraarterial bolus injections of adenosine on heart rate $(H R, t o p)$, mean arterial blood pressure (MABP, middle), and muscle sympathetic nerve activity (MSNA, bottom). Data are expressed as changes $(\Delta)$ or percent changes $(\% \Delta)$ from the preceding resting period. Measurements were performed during the period from 30 to $90 \mathrm{~s}$ after injections. Drugs were administered into the brachial artery during simultaneous venous occlusion of the forearm. $n=6 ; P$ values are those from ANOVA; asterisks indicate significant differences compared with saline, using post hoc tests (Duncan's).

was accompanied by an increase in heart rate and MSNA. All of these effects were over in $60 \mathrm{~s}$. Identical effects were observed after bolus injections of adenosine into the brachial artery (data not shown), implying that, at these doses, intrabrachial adenosine reaches the systemic circulation. A different pattern of effects was observed when adenosine was injected into the brachial artery during simultaneous venous occlusion; blood pressure and MSNA increased gradually and in parallel, whereas minute ventilation did not change significantly.

The dose-response curve of these changes are shown in Fig. 2. Intraarterial bolus injections of adenosine during simultaneous venous occlusion produced a dose-dependent increase in MSNA ( $n=6, P<0.01$ by ANOVA) and in blood pressure ( $P$ $<0.01)$. Heart rate also increased $(P=0.03)$ but only at the highest dose of adenosine. Adenosine (4 mg) increased MSNA by $96 \pm 25 \%$ and raised mean arterial blood pressure by $12 \pm 3$ mmHg. The data presented in Fig. 2 were obtained by averaging heart rate and blood pressure values at the time of peak effects of each bolus of saline or adenosine, and by integrating MSNA over $1 \mathrm{~min}$, starting $30 \mathrm{~s}$ after drug administration.

Adenosine produced a greater increase in MSNA than cold exposure or ischemia, when both stimuli were paired to produce the same intensity of forearm discomfort (Fig. 3). 

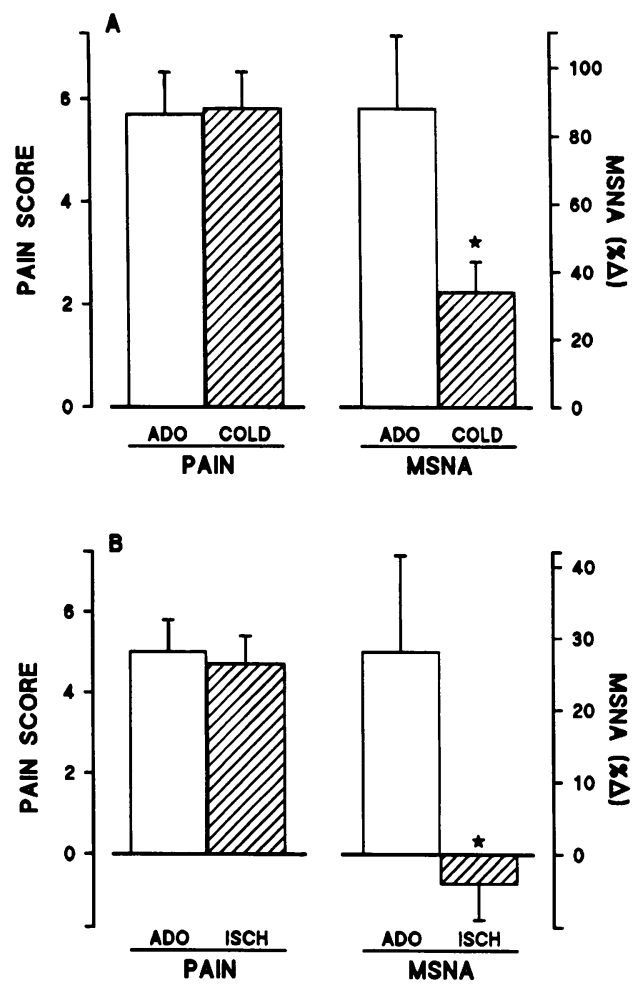

Figure 3. Comparison of the effects of intrabrachial adenosine to other painful stimuli. $(A)$ Comparison of the effects of intrabrachial adenosine $(A D O, 2.7 \pm 0.6 \mathrm{mg}$, open bars, $n=6)$ and cold exposure (COLD, hatched bars) on pain score (left bars, maximal pain score $=10)$ and muscle sympathetic nerve activity (MSNA, right bars, expressed as the percent change form the preceding baseline period). $(B)$ Comparison of the effect of intrabrachial adenosine ( $A D O$, $1.8 \pm 0.5 \mathrm{mg}$, open bars, $n=6$ ) and forearm ischemia (ISCH, hatched bars, duration of ischemia $=280 \pm 20 \mathrm{~s}$ ). In both cases stimuli were titrated to produce a similar level of forearm pain in the same individual. Other methods are similar to those of Fig. 2.

Effect of adenosine receptor blockade with theophylline on the exercise pressor reflex. Intrabrachial infusion of theophylline produced small changes in heart rate, blood pressure, and MSNA that were not different from those produced by saline (Table I). Venous forearm samples taken at the end of theophylline infusion revealed theophylline plasma levels of $5.1 \pm 1.1$ $\mu \mathrm{g} / \mathrm{ml}(28 \pm 6 \mu \mathrm{M})$ in the infused arm. Plasma levels in the contralateral arm, reflecting the degree of systemic exposure to theophylline, were $1.0 \pm 0.2 \mu \mathrm{g} / \mathrm{ml}(5 \pm 1 \mu \mathrm{M})$.

Static isometric exercise produced a significant increase in MSNA that progressed throughout the duration of the handgrip and reached a maximal increase of $92 \pm 15 \%$ during the third minute. MSNA remained above baseline during the period of post-handgrip ischemia (Fig. 4). Similar time-course changes in blood pressure and heart rate were observed. The increase in MSNA produced by isometric exercise was significantly inhibited during intrabrachial administration of theophylline $(92 \pm 15 \%$ vs. $28 \pm 6 \%$ during the third minute of handgrip), and was completely abolished during the period of posthandgrip ischemia (Fig. 4; $n=7, P<0.01$ ). The increase in blood pressure produced by handgrip was also blunted by intrabrachial theophylline $(P=0.01,38 \pm 6$ vs. $27 \pm 4 \mathrm{mmHg}$ during the third minute of handgrip). On the other hand, theophyl-
Table I. Effect of Intrabrachial Infusion of Saline or Theophylline on Baseline Parameters

\begin{tabular}{lcc}
\hline & Saline & Theophylline \\
\hline$\Delta$ HR (bpm) & $-1 \pm 1.4$ & $-2 \pm 1$ \\
$\Delta$ SBP (mm Hg) & $+6 \pm 3$ & $+3 \pm 2$ \\
$\Delta$ DBP (mm Hg) & $+9 \pm 2$ & $-4 \pm 3$ \\
$\Delta$ MSNA (\%) & $+47 \pm 6$ & $+33 \pm 10$ \\
\hline
\end{tabular}

line had no significant effect on the changes in heart rate produced by isometric exercise.

Isometric exercise was repeated during simultaneous circulatory arrest of the exercising muscle in an attempt to provide a more potent metabolic stimulus. Isometric exercise during circulatory arrest produced a greater increase in muscle sympathetic nerve activity than isometric exercise alone. MSNA increased by $77 \pm 16 \%$ during the second minute of isometric exercise and by $173 \pm 39 \%$ during the second minute of isometric exercise during circulatory arrest. The increase in MSNA produced by isometric exercise during circulatory arrest was blunted by intrabrachial theophylline to $44 \pm 19 \%$ during the second minute (Fig. $5 ; n=7, P<0.01$ by ANOVA). Theophylline also inhibited the increase in mean arterial blood pressure produced by isometric handgrip during circulatory arrest ( $45 \pm 7$ vs. $27 \pm 1 \mathrm{mmHg}$ during the second minute of handgrip), although this difference did not reach statistical significance $(P$
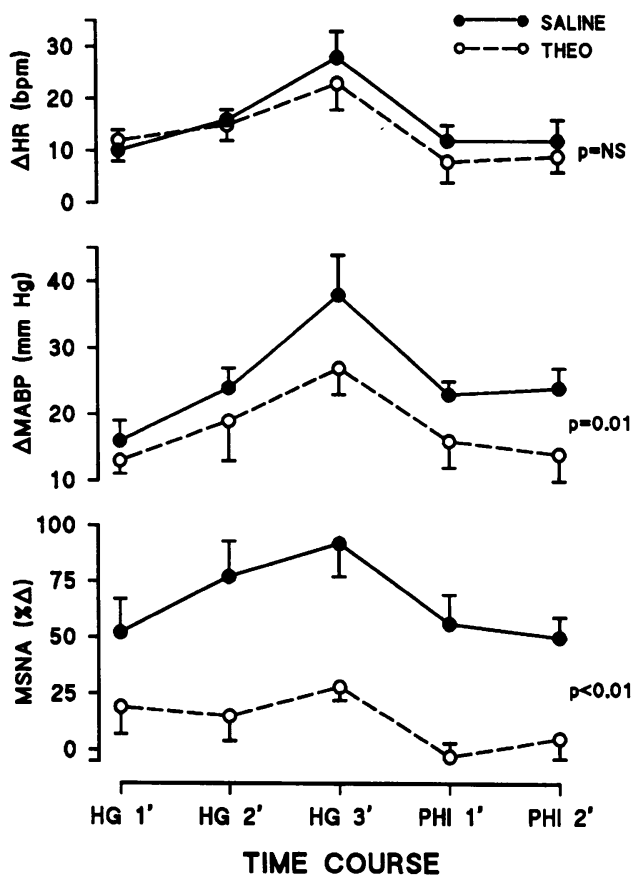

Figure 4. Effect of intrabrachial theophylline on the cardiovascular and sympathetic response to isometric exercise. Line graphs show the effects of 3 min of sustained handgrip ( $H G, 30 \%$ of maximal voluntary contraction) followed by 2 min of posthandgrip ischemia (PHI, circulatory arrest of the forearm during the release of handgrip). Procedures were repeated during infusion of saline $(\bullet)$ or theophylline $(\mathrm{O}$, $1 \mu \mathrm{g} / \mathrm{ml}$ forearm $/ \mathrm{min}$ ). $n=6 ; P$ values are from analysis of variance for the overall comparison between saline and theophylline. Other methods and abbreviations are similar to those of Fig. 2. 
$=0.06$ by ANOVA). The increase in heart rate produced by isometric handgrip during circulatory arrest was unaffected by theophylline.

We thought it important to use each subject as his own control because of the interindividual variability in the magnitude of sympathetic activation produced by the exercise pressor reflex. For this reason, the saline infusion period preceded the theophylline infusion period in all subjects. The order of infusions could not be reversed because of the half-life of theophylline. Responses to isometric exercise of the right hand were used as a control to account for possible time-related effects and for potential systemic effects of intrabrachial theophylline. Intrabrachial infusion of theophylline into the left arm did not alter the increase in MSNA produced by isometric exercise of the right arm (Fig. 6).

Isometric handgrip of the left arm produced a greater increase in MSNA than isometric handgrip of the right arm. It has previously been shown that exercise of the dominant, and therefore, more conditioned arm elicits a lesser increase in MSNA than exercise of the nondominant arm (12). Our results during saline administration can be explained by this mechanism because all the volunteers participating in this protocol study were right handed.

\section{Discussion}

The specific metabolic events involved in the exercise pressor reflex are not completely understood. It has been proposed that

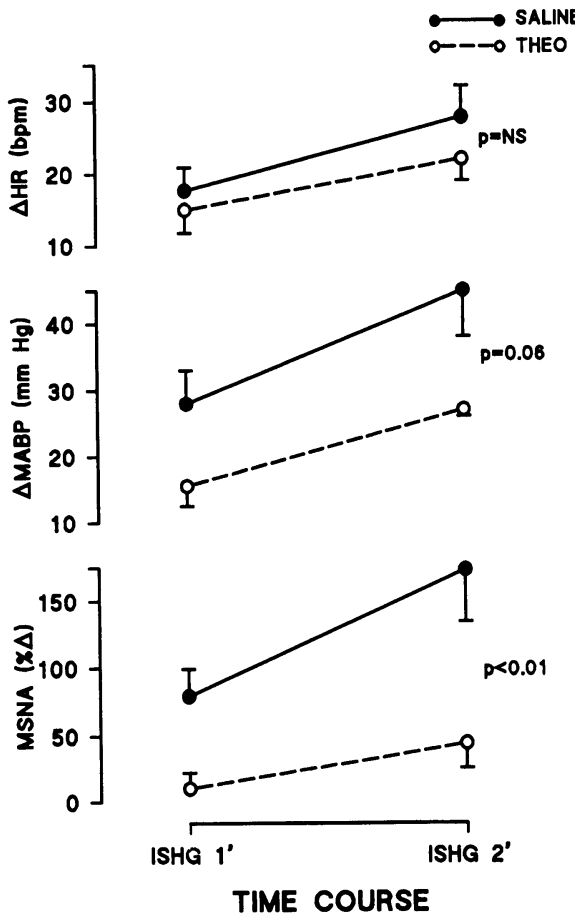

Figure 5. Effect of intrabrachial theophylline on the cardiovascular and sympathetic response to isometric exercise during simultaneous forearm ischemia. Line graphs show the effects of $2 \mathrm{~min}$ of sustained handgrip ( $30 \%$ of maximal voluntary contraction) during simultaneous circulatory arrest of the forearm (ischemic handgrip, ISHG). Procedures were repeated during infusion of saline $(\bullet)$ or theophylline $(0,1 \mu \mathrm{g} / \mathrm{ml}$ forearm). $n=7 ; P$ values are from analysis of variance for the overall comparison between saline and theophylline. Other methods and abbreviations are similar to those of Fig. 2.

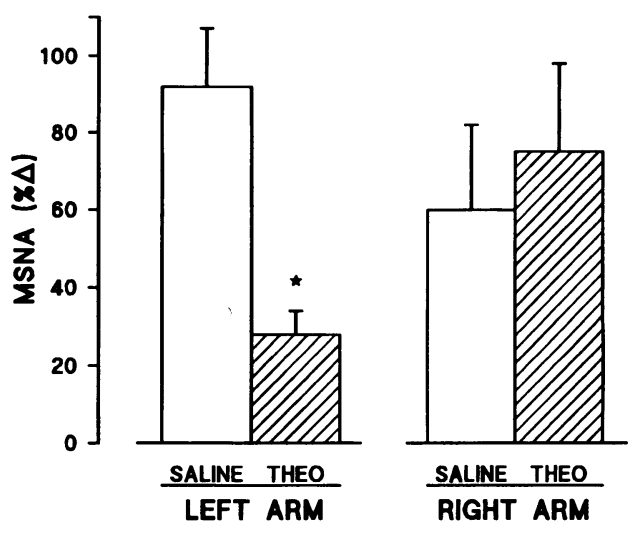

Figure 6. Bar graph shows the increase in muscle sympathetic nerve activity ( $M S N A$, expressed as the percent change from the preceding resting period) produced during the third minute of isometric handgrip (30\% of maximal voluntary contraction) of the left or right arm during infusion of saline or theophylline (THEO) into the left brachial artery. Asterisk denotes significant difference compared to saline using $t$ test; $n=5$.

hydrogen ions are involved in muscle afferent activation. In favor of this proposal, it was found that a decrease in muscle intracellular $\mathrm{pH}$ parallels the increase in muscle sympathetic activity in response to isometric exercise $(13,14)$. Furthermore, muscle contraction in patients with muscle phosphorylase deficiency (McArdle's disease) is not accompanied by glycogen degradation or a decrease in muscle $\mathrm{pH}$, and MSNA does not increase in response to isometric exercise in these patients (15). It has been argued, however, that other metabolic products may also play a role in this reflex. For example, exercise of a nonconditioned muscle produces a greater increase in MSNA than exercise of a conditioned muscle, even though a similar decrease in muscle $\mathrm{pH}$ is produced in both cases (12), suggesting that the decrease in muscle $\mathrm{pH}$ is not the sole mediator of this reflex. However, other potential metabolic mediators responsible for the triggering of the exericse pressor reflex have not been previously identified.

Adenosine can be proposed as a likely candidate because it is known to be produced in skeletal muscles during increased metabolic demands and ischemia (2). There is extensive evidence, however, that adenosine acts as an inhibitory neuromodulator. Adenosine hyperpolarizes neurons, decreases nerve firing, prevents seizures, and inhibits neurotransmitter release $(16,17)$. These inhibitory effects are particularly prominent in efferent nerves and in the central nervous system. Even in the central nervous system, however, adenosine may have excitatory actions, enhancing the release of glutamate in the nucleus of the solitarii tract (18) and in hippocampal neurons (19). On the other hand, adenosine has prominent excitatory actions in afferent nerves. To the best of our knowledge, adenosine activates all afferents so far examined, including renal and myocardial afferents, and arterial chemoreceptors. We, therefore, hypothesized that adenosine would also activate the muscle afferents involved in the exercise pressor reflex.

In support of this hypothesis, we previously found that intrabrachial injections of adenosine produced a modest but significant increase in MSNA (20). An increase in blood pressure was also observed, but this effect was not significant at the relatively low doses used $(0.5-1.5 \mathrm{mg})$. In the present study we were able to administer higher doses by occluding the venous 
circulation of the forearm to avoid significant spillover of intraarterial adenosine into the systemic circulation. This approach is possible because of the extremely short half-life of adenosine in blood, reportedly $<1 \mathrm{~s}$. (21). The effectiveness of this strategy is evidenced by the lack of ventilatory stimulation, adenosine's most consistent and dramatic systemic effect, when it was administered intraarterially during venous occlusion.

Using this approach, we found that intrabrachial adenosine produced gradual and parallel increases in MSNA and blood pressure at doses that had no effect on heart rate ( 2 and $3 \mathrm{mg}$ ). Isometric exercise, by comparison, also increases heart rate in addition to increasing blood pressure and MSNA. The increase in heart rate produced by voluntary exercise, however, is largely due to the voluntary central command initiating the exercise, rather than to activation of muscle afferents (22). The effects of intrabrachial adenosine, therefore, mimic those expected to result from the selective activation of muscle afferents by isometric exercise.

Before we can conclude that adenosine indeed activates muscle afferents in the forearm, alternative explanations to our findings need to be considered. Adenosine is a potent vasodilator in the forearm. It could be proposed, therefore, that the systemic sympathetic activation produced by intrabrachial adenosine is due to local vasodilatation. This seems unlikely because we have previously shown that intrabrachial nitroprusside, administered at a dose titrated to produce the same magnitude of forearm vasodilation as adenosine, has no effect on MSNA (20). Intrabrachial adenosine also produces forearm discomfort (8), possibly through activation of sensory afferents (23) that share the morphological and electrophysiological characteristics of muscle sympathetic afferents (slow-conducting group IV C-fibers) (24). The increase in MSNA produced by intrabrachial adenosine, however, was greater than that produced by cold or ischemia when these stimuli were titrated to produce a comparable intensity of pain. Therefore, activation of sensory afferents may contribute to, but does not completely explain, the increase in MSNA produced by intrabrachial adenosine.

Two limitations of this first set of experiments should be considered. First, it remains possible that adenosine activates other as yet undetermined structures in the forearm, leading to sympathetic activation. From the results obtained using exogenous adenosine, therefore, we cannot definitely conclude that adenosine activates muscle afferents. Second, it can be argued that we used supraphysiological doses of adenosine. It is likely, however, that most of the intraarterially injected adenosine is trapped by the endothelium because of the highly effective nucleoside uptake system, and very little reaches the interstitium (25), our putative site of action. It is, therefore, impossible to estimate how much of the adenosine injected intraarterially actually reaches the muscle interstitium, and whether such concentrations constitute "supraphysiological" levels.

Because of these limitations and as a complementary approach to this problem, we also determined if blocking endogenous adenosine may alter the cardiovascular and neural responses to the exercise pressor reflex. For this purpose we administered the adenosine receptor antagonist theophylline intrabrachially $(26,27)$ at doses that lacked significant systemic effects but were sufficient to antagonize the increase in MSNA produced by exogenous adenosine, while the exercise pressor reflex was evoked by isometric handgrip. Intrabrachial theophylline blunted the increase in MSNA and mean arterial blood pressure produced by isometric exercise, but had no effect on the increase in heart rate. Similar effects were observed if a greater metabolic stimuli was induced by performing exercise during circulatory arrest of the forearm. The fact that intrabrachial theophylline selectively blocks the sympathetic activation and the increase in blood pressure produced by exercise, but not the increase in heart rate, supports the hypothesis that endogenous adenosine contributes to muscle afferent activation. As mentioned previously, the increase in heart rate during isometric exercise is to a large extent due to the voluntary central command initiating the exercise rather than to muscle afferent activation.

We also must consider potential limitations of this second set of experiments. First, theophylline seemed to produce a greater inhibition of the increase in MSNA than the increase in blood pressure produced by isometric exercise. Several reasons may account for this apparent difference. Theophylline did not blunt the exercise-induced tachycardia, which may contribute to the pressor effect by increasing cardiac output. Likewise, if our hypothesis is correct, intrabrachial theophylline would selectively block adenosine-induced muscle afferent activation, but will have no effect on central command or muscle mechanoreceptors, which may increase blood pressure through mechanisms that are not reflected in MSNA. Second, it could be argued that theophylline may have other nonspecific effects, such as inhibition of phosphodiesterase. The potency of theophylline as a phosphodiesterase inhibitor $\left(\mathrm{IC}_{50}\right.$ of $0.1-0.2 \mathrm{mM}$ $[28,29])$ is much lower than as an adenosine receptor antagonist ( $\mathrm{IC}_{50}$ of 2-50 $\mu \mathrm{M}$ [30]). By comparison, the plasma concentration of theophylline obtained in the infused arm was $28 \pm 6 \mu \mathrm{M}$. Third, it could be argued that the effect of theophylline on the exercise pressor reflex was due to a systemic effect rather than local inhibition of adenosine in the exercising forearm. Systemic plasma levels of theophylline, however, were significantly lower $(5 \pm 1 \mu \mathrm{M})$ than those considered therapeutically effective (20-50 $\mu \mathrm{M})$. More importantly, theophylline had no effect on the exercise pressor response to exercise of the contralateral arm, implying that theophylline had no significant systemic effects. These results also imply that the differences in response to exercise of the theophylline-infused arm were not due to time-related effects. This is an important consideration given the sequential design of our study (saline followed by theophylline).

The results of our experiments, taken together, suggest that adenosine contributes to the sympathetic activation produced by isometric exercise in humans. It must be noted that Roto and Kauffman (31) found that adenosine and its analogue, 2-choloroadenosine, stimulated only $15 \%$ of muscle afferents studied in an isolated feline lower limb preparation. They concluded that adenosine did not significantly stimulate muscle afferents. Indirect evidence, however, suggests that adenosineinduced activation of sympathetic afferents is particularly prominent in humans. For example, intravenous adenosine produces a dramatic hypotension in virtually all animal models without the adequate sympathetic activation expected for a vasodilator $(32,33)$. This lack of reflex sympathetic activation is probably explained by the inhibitory actions of adenosine. In contrast, intravenous adenosine produces sympathetic activation in conscious humans (7), probably by stimulation of arterial chemoreceptor afferents (11). This effect is of such magnitude in humans that it overwhelms the vasodilatory, bradycardic, and neuroinhibitory actions of adenosine that are so 
prominent in other animal species. It is possible, therefore, that the apparent contradiction between our findings and those reported in cats are explained by species differences. Important species differences have also been reported for other actions of adenosine (34).

It has been proposed that the primary role of adenosine is to maintain local homeostasis by counteracting excessive metabolic demands on the cell. It is generally considered that adenosine fulfills this role through its inhibitory actions (e.g., vasodilation, inhibition of norepinephrine release). The excitatory action of adenosine reported here would appear to be at odds with this role. However, by activating muscle afferents, adenosine will produce systemic sympathetic activation and increase blood pressure, which will result in improved perfusion pressure to the exercising muscle. At the same time adenosine will act locally to inhibit norepinephrine release and to produce vasodilatation, thus protecting the exercising muscle from the increased sympathetic activity.

In conclusion, we postulate adenosine as one of the metabolic products that contribute to the activation of muscle afferents involved in the exercise pressor reflex in humans. Furthermore, we speculate that these excitatory effects of adenosine works in tandem with its inhibitory actions to protect the exercising muscle.

\section{Acknowledgments}

The authors would like to thank Dr. David Robertson for his input in the formulation of the hypotheses underlying these studies, and Mrs. Jane Estrada and Dorothea Boemer for editorial assistance.

This work was supported in part by grants HL-14192 (Specialized Center of Research in Hypertension) and RR-00095 (Clinical Research Center) from the National Institutes of Health, and a grant from the Life Sciences Institute.

\section{References}

1. Mark, A. L., R. G. Victor, C. Nerhed, and B. G. Wallin. 1985. Microneurographic studies of the mechanisms of sympathetic nerve responses to static exercise in humans. Circ. Res. 57:461-469.

2. Ballard, H. J. 1991. The influence of lactic acid on adenosine release from skeletal muscle in anesthetized dogs. J. Physiol. 433:95-108.

3. Biaggioni, I. 1992. Contrasting excitatory and inhibitory effects of adenosine in blood pressure regulation. Hypertension (NY). 20:457-465.

4. Katholi, R. E., G. R. Hageman, P. L. Whitlow, and W. T. Woods. 1983. Hemodynamic and afferent renal nerve responses to intrarenal adenosine in the dog. Hypertension (NY). 5(Suppl. I):149-154.

5. Cox, D. A., J. A. Vita, C. B. Treasure, R. D. Fish, A. P. Selwyn, and P. Ganz. 1989. Reflex increase in blood pressure during the intracoronary administration of adenosine in man. J. Clin. Invest. 84:592-596.

6. Runold, M., N. S. Cherniack, and N. R. Prabhakar. 1990. Effect of adenosine on isolated and superfused cat carotid body activity. Neurosci. Lett. 113:111114.

7. Biaggioni, I., T. J. Killian, R. Mosqueda-Garcia, R. M. Robertson, and D. Robertson. 1991. Adenosine increases sympathetic nerve traffic in humans. Circulation. 83:1668-1675.

8. Sylven, C., B. Jonzon, B.. Fredholm, and L. Kaijser. 1988. Adenosine injections into the brachial artery produces ischemia like pain or discomfort in the forearm. Cardiovasc. Res. 22:674-678.

9. Taddei, S., R. Pedrinelli, and A. Salvetti. 1991. Theophylline is an antagonist of adenosine in human forearm arterioles. Am. J. Hypertens. 4:256-259.

10. Biaggioni, I., S. Paul, and D. Robertson. 1988. A simple high pressure liquid chromatographic method applied to determine caffeine in plasma and tissues. Clin. Chem. 34:2345-2348.

11. Biaggioni, I., B. Olafsson, R. M. Robertson, A. S. Hollister, and D. Robertson. 1987. Cardiovascular and respiratory effects of adenosine in conscious man: Evidence for chemoreceptor activation. Circ. Res. 61:779-786.

12. Sinoway, L. I., R. F. Rea, T. J. Mosher, M. B. Smith, and A. L. Mark. 1992. Hydrogen ion concentration is not the sole determinant of muscle metaboreceptor responses in human. J. Clin. Invest. 89:1875-1884.

13. Victor, R. G., L. A. Bertocci, S. L. Pryor, and R. L. Nunnally. 1988. Sympathetic nerve discharge is coupled to muscle cell pH during exercise in humans. J. Clin. Invest. 82:1301-1305.

14. Rotto, D. M., C. L. Stebbins, and M. P. Kaufman. 1989. Reflex cardiovascular and ventilatory responses to increasing $\mathrm{H}+$ activity in cat hindlinb muscle. J. Appl. Physiol. 67:256-263.

15. Pryor, S. L., A. F. Lewis, R. G. Haller, L. A. Bertocci, and R. G. Victor. 1990. Impairment of sympathetic activation during static exercise in patients with muscle phosphorylase deficiency (McArdle's disease). J. Clin. Invest. 85:1444-1449.

16. Phillis, J. W., and P. H. Wu. 1981. The role of adenosine and its nucleotides in central synaptic transmissions. Prog. Neurobiol. (NY). 16:187-239.

17. Wakade, A. R., and T. D. Wakade. 1978. Inhibition of noradrenaline release by adenosine. J. Physiol. 282:35-49.

18. Mosqueda-Garcia, R., C-J. Tseng, M. Appalsamy, C. Beck, and D. Robertson. 1991. Cardiovascular excitatory effects of adenosine in the nucleus of the solitary tract. Hypertension (NY). 18:494-502.

19. Sakurai, T., and Y. Okada. 1992. Excitatory effect of adenosine on neurotransmission and the release of glutamate from hippocampal slices of guinea-pig. J. Physiol. 446:P380.

20. Costa, F., and I. Biaggioni. 1993. Adenosine activates afferent fibers in the forearm, producing sympathetic stimulation in humans. J. Pharmacol. Exp. Ther. 267:1369-1374.

21. Moser, G. H., J. Schrader, and A. Deussen. 1989. Turnover of adenosine in plasma of human and dog blood. Am. J. Physiol. 256:C799-C806.

22. Victor, R. G., D. R. Seals, and A. L. Mark. 1987. Differential control of heart rate and sympatheic nerve activity during dynamic exercise. J. Clin. Invest. 79:508-516.

23. Bleehan, T., and C. A. Keele. 1977. Observations on the algogenic actions of adenosine compounds on the human blister base preparation. Pain. 3:367377.

24. Mitchell, J. H., M. P. Kaufman, and G. A. Iwamoto. 1983. The exercise pressor reflex: its cardiovascular effects, afferent mechanisms, and central pathways. Annu. Rev. Physiol. 45:229-242.

25. Nees, S., V. Herzog, B. F. Becker, M. Bock, C. Des Rosiers, and E. Gerlach. 1985. The coronary endothelium: a highly active metabolic barrier for adenosine. Basic Res. Cardiol. 80:515-529.

26. Smits, P., J. W. M. Lenders, and T. Thien. 1990. Caffeine and theophylline attenuate adenosine-induced vasodilatation in humans. Clin. Pharmacol. \& Ther. 48:410-418.

27. Biaggioni, I., S. Paul, A. Puckett, and C. Arzubiaga. 1991. Caffeine and theophylline as adenosine receptor antagonists in humans. J. Pharmacol. Exp. Ther. 258:588-593.

28. Kramer, G. L., and J. N. Wells. 1979. Effects of phosphodiesterase inhibitors on cyclic nucleotide levels and relaxation of pig coronary arteries. Mol. Pharmacol. 16:813-822.

29. Rall, T. W. 1982. Evolution of the mechanisms of action of methylxanthines: From calcium mobilizers to antagonists of adenosine receptors. Pharmacologist. 24:277-287.

30. Feoktistov, I., and I. Biaggioni. 1993. Characterization of A2 adenosine receptors in human erythroleukemia cells and in platelets. Mol. Pharmacol. 43:909-914.

31. Rotto, D. M., and M. P. Kaufman. 1988. Effect of metabolic products of muscular contraction on discharge of group III and IV afferents. J. Appl. Physiol. 64:2306-2313.

32. Delle, M., S. E. Ricksten, and D. Delbro. 1988. Pre- and postganglionic sympathetic nerve activity during induced hypotension with adenosine or sodium nitroprusside in the anesthetized rat. Anesth. Analg. 67:307-312.

33. Ohnishi, A., I. Biaggioni, G. Deray, R. A. Branch, and E. K. Jackson. 1986. Hemodynamic effects of adenosine in conscious hypertensive and normotensive rats. Hypertension (NY). 8:391-398.

34. Froldi, G., and L. Belardinelli. 1990. Species-dependent effects of adenosine on heart rate and atrioventricular nodal conduction. Circ. Res. 67:960-978. 